\title{
Precession electron diffraction \& automated crystallite orientation/phase mapping in a transmission electron microscope
}

\author{
Peter Moeck ${ }^{1}$, Sergei Rouvimov ${ }^{1}$, Ines Häusler ${ }^{2,1}$, Wolfgang Neumann ${ }^{3,2}$, and Stavros Nicolopoulos ${ }^{4}$ \\ ${ }^{1}$ Nano-Crystallography Group, Department of Physics, Portland State University, Portland, OR 97207-0751 \& Oregon \\ Nanoscience and Microtechnologies Institute \\ ${ }^{2}$ Institute of Physics, Humboldt University of Berlin, Newtonstraße 15, 12489 Berlin, Germany \\ ${ }^{3}$ Department of Chemistry and Center for Green Materials Chemistry, 1252 University of Oregon, Eugene OR 97403 \\ ${ }^{4}$ NanoMEGAS SPRL, Boulevard Edmond Machterns No 79, Saint Jean Molenbeek, Brussels, B-1080, Belgium \\ Email: rouvimov@pdx.edu,pmoeck@pdx.edu, haeusler@physik.hu-berlin.de, info@ nanomegas.com
}

\begin{abstract}
The basics of precession electron diffraction (PED) in a transmission electron microscope (TEM) are briefly discussed. An automated system for the mapping of nanocrystal phases and orientations in a TEM is briefly described. This system is primarily based on the projected reciprocal lattice geometry as extracted from experimental precession electron diffraction spot patterns. Comprehensive open-access crystallographic databases may be used in support of the automated crystallite phase identification process and are, therefore, also briefly mentioned.
\end{abstract}

Index Terms - Nanometrology, Precession Electron Diffraction, Transmission Electron Microscopy

\section{INTRODUCTION}

An automated electron diffraction system for orientation and phase mapping in the TEM has been demonstrated for the first time [1] in the fall of 2008. Precession electron diffraction (PED) [2] proved crucial to the success of the system [3]. The Brussels/Belgium based NanoMEGAS company offers a turn-key solution to automated PED based nanocrystal phase and orientation mapping in the TEM.

This paper consists of four sections. We start with an introduction to PED and follow up with a brief overview over NanoMEGAS' system. Typical results of the application of this system are then shown. These results are taken from previous experimental studies which were performed both in Portland/Oregon and Berlin/Germany. Comprehensive open-access crystallographic databases that may be used in support of the mapping system's software are mentioned in the final section.

\section{BASICS OF PRECESSION ELECTRON DIFFRACTION}

The (single-crystallite) PED technique is formally analogous to the well known (single-crystal) X-ray Buerger precession technique. It utilizes, however, a precession movement of the primary electron beam around the microscope's optical axis while the crystalline specimen remains stationary, Fig. 1. This is equivalent to an Ewald sphere that is precessing around the TEM's optical axis, Fig. 2. All of the advantages of PED for structural fingerprinting [4-6], electron crystallography of nanocrystals, and automated crystallite orientation and phase mapping can be illustrated on the basis of this concept [3].

The precession angle $(\varepsilon)$ is in PED at most a few degrees, i.e. on the order of the electron diffraction angles $(2 \theta)$. The primary electron beam can be either parallel or slightly convergent and its precession creates a hollow illumination cone which has its vertex on the crystalline sample. After they have left the exit face of the crystalline sample, the transmitted electron beam and the diffracted beams are "descanned" (un-precessed) in such a manner that stationary electron diffraction spot patterns are obtained on the diffraction plane (and also on the viewing screen of the TEM), Fig. 1 illustrates both the hollow cone illumination and proper un-precessing of a diffracted beam and the transmitted beam.

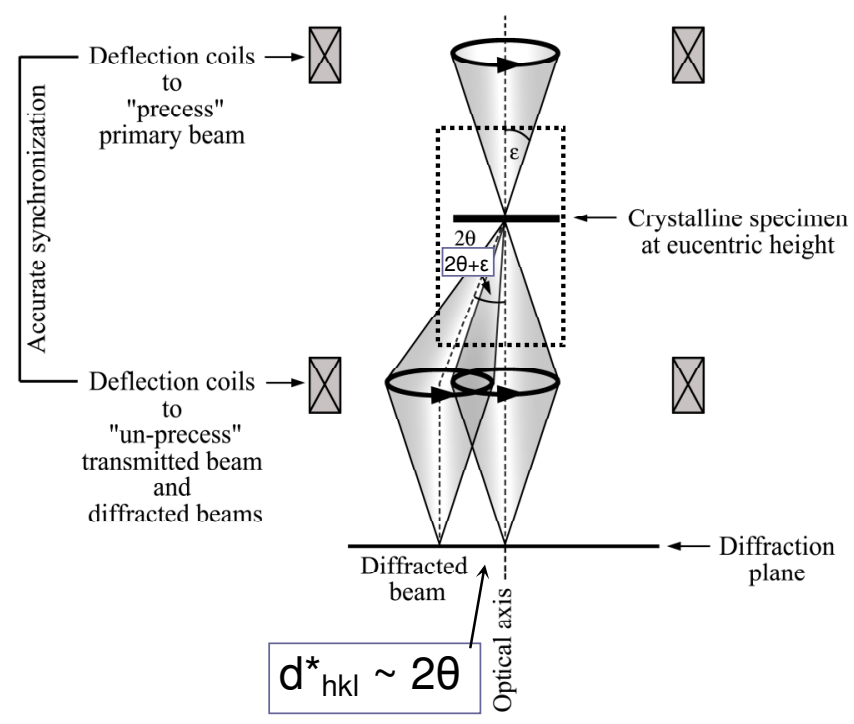

Fig. 1 Direct space representation of the PED mode. The dash-boxed area around the specimen is shown in reciprocal space in Fig. 2. 
The nature of the crystalline sample, i.e. it being either a single crystallite with a size in the tens of nanometers range or a multitude of crystallites, determines the recorded PED pattern as being either of the single crystallite spot pattern type or the powder ring pattern type. The powder kind of PED can be thought of as consisting of a multitude of superimposed single crystallite PED patterns.

It is instructive to compare both kinds of PED patterns to ordinary parallel illumination, stationary primary electron beam selected area electron diffraction (SAED) and ("parallel due to a small condenser aperture") nano-beam electron diffraction (NBED) patterns. In both cases, the PED patterns possess more reflections than the corresponding SAED or NBED patterns and contain additional quantifiable crystal structure information. One may explain this enhanced structural information content as being caused by the superposition of a multitude of individual sequentially recorded diffraction patterns that differ from each other by their primary electron beam direction within the hollow illumination cone of the PED geometry, Figs. 1 and 2.

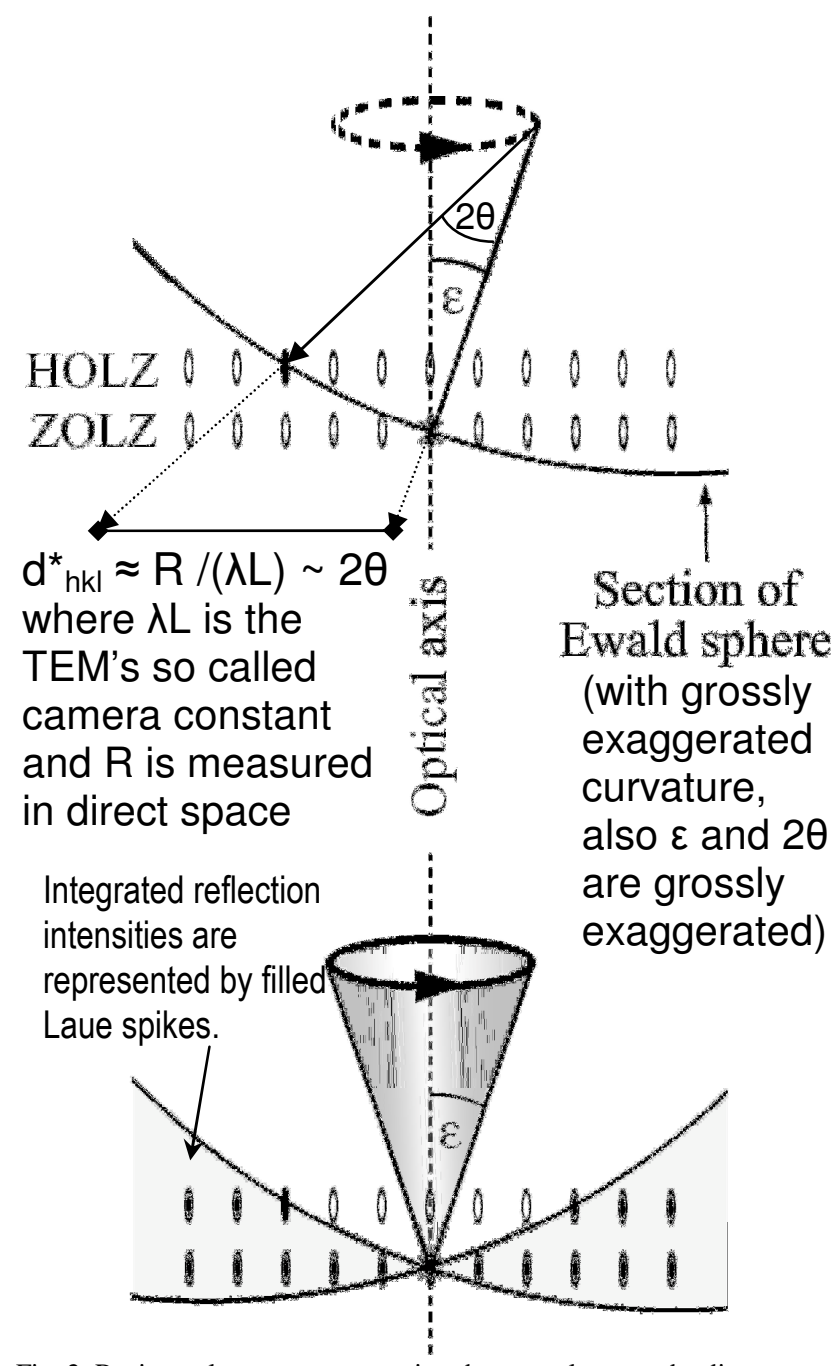

Fig. 2 Reciprocal space representation that complements the direct space representation of the PED mode within the dash-boxed area of Fig. 1.

Assuming a single crystallite sample oriented with a low indexed zone axis more or less parallel to the optical axis of the TEM, the transmitted electron beam direction of each of these patterns needs to be indexed with rather high Miller indices. As a consequence, reflections from higher order Laue zones (HOLZs) may be present in a PED spot pattern in addition to reflections from the zero order Laue zone (ZOLZ). It is well known that the lattice constant parallel to the optical axis of the TEM can be extracted from the position of HOLZ reflections in an electron diffraction spot pattern.

As the upper part of Fig. 2 illustrates, there is a chance that only a few beams are excited at any one time during a precession cycle so that dynamical diffraction effects may be effectively suppressed. This may results in "quasi-kinematic" reflection intensities if the crystallite is sufficiently thin (its unit cell sufficiently large, and the electron wavelength sufficiently short). Also kinematically forbidden reflections will be suppressed effectively, especially at large precession angles, since there are much fewer double diffraction routes.

A full precession cycle, see lower part of Fig. 2, results for many reflection in a more or less full integration of their intensity over the excitation error. PED patterns of single crystallites display, therefore, the projection symmetry of the reciprocal lattice as long as the misorientation of the crystallites with respect to the optical axis is significantly smaller than the precession angle.

\section{Automated CRYSTALLITE ORIENTATION AND PHASE MAPPING IN THE TEM}

NanoMEGAS' automated technique for the mapping of crystallite orientations and phases of polycrystalline materials in the TEM has so far been utilized by us for a variety of crystallographic analyses [3,7-9, and references therein]. This technique benefits greatly from PED and is based on template matching of experimental electron diffraction spot patterns to their pre-calculated theoretical counterparts, as obtained from the kinematical diffraction theory. It relies, therefore, mainly on the projected reciprocal lattice geometry and partly on the reflection's intensities. Figure 3 shows a sketch/photograph of the whole ASTAR/DigiSTAR system as interfaced to a generic TEM.

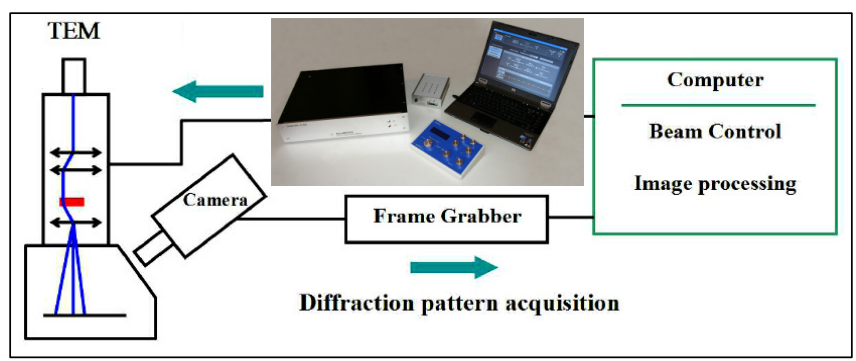

Fig. 3. Experimental set up of the ASTAR/DigiSTAR system for automated crystallite orientation and phase mapping, modified after ref. [3] The system consists of hard- and software. The hardware can be interfaced to any older or newer mid-voltage TEM. The software can read

Crystallographic Information Files that are freely downloadable from comprehensive open-access crystallographic databases.

PED patterns are automatically recorded by an external digital camera while the area of interest is scanned in a step 
like fashion with a nanometer to tens of nanometers diameter primary electron beam. For mapping purposes, precession angles between $0.1^{\circ}$ to $1^{\circ}$ are typically sufficient. The precession angle, the number of simulated templates, and the often unavoidable thin film buckling affect the maximal obtainable orientation resolution, which is typically better than $1^{\circ}$ degree. The data collection step is followed by semiautomatic data processing.

Figure 4 illustrates in its first column schematically the ASTAR/DigiSTAR settings PED “off" and "on". The second column of this figure shows images of the transmitted electron beam (without a sample) for precession angles of zero (i.e. "PED off") and $0.96^{\circ}$. Since the selection of the sampled area is done directly with the primary/transmitted electron beam, it is important that a very small, but nearly parallel electron beam can be formed in the first place and maintained during precession.

The electron probes that are shown in the second column of Fig. 4 were obtained at the JEOL JEM 2200FS of Humboldt University Berlin, which possesses a fieldemission gun, a parallel nanoprobe mode, and an in-column $\Omega$ energy filter. A $10 \mu \mathrm{m}$ diameter aperture in the second condenser lens can be used in this microscope in order to obtain a non-precessing electron probe of less than $3 \mathrm{~nm}$ on a routine basis. Precession at about $1^{\circ}$ leads typically to a five to ten fold increase of the effective size of the (nearly parallel) nanoprobe, Fig. 4. Many modern field emission gun microscopes possess similar capabilities of forming (nearly) parallel nanoprobes with nanometer or sub-nanometer diameters, e.g. ref. [10].
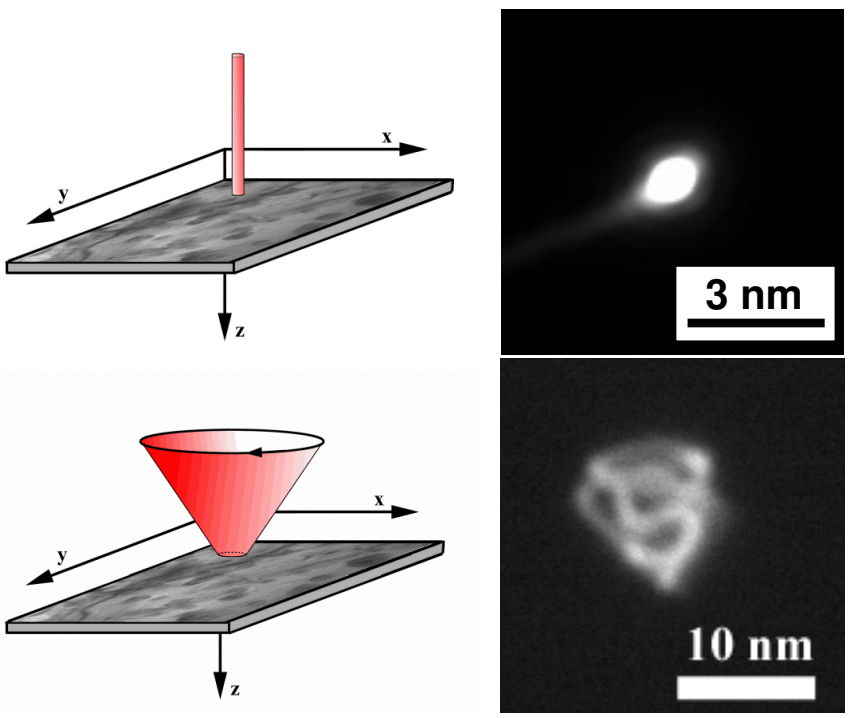

Fig. 4. Schematic sketch of the ASTAR/DigiSTAR settings PED "off" and "on" (first column) and corresponding sizes of the transmitted electron beam in the parallel nanoprobe mode (second column) as obtainable with modern field emission gun microscopes. The precession angle in the sketch is grossly exaggerated.

\section{ORIENTATION DISTRIBUTION IN POLYCRYSTALLINE COPPER}

Thin polycrystalline copper films were analyzed at the FEI Tecnai $\mathrm{G}^{2}$ F20 ST analytical field-emission microscope of Portland State University at $200 \mathrm{kV}$ [3]. This microscope is equipped with NanoMEGAS' ASTAR/DigiSTAR system and serves as demo site for this company for North America.

Due to its field emission source, nanometer sized transmitted beams with very small convergence angels can be utilized in this microscope in conjunction with suitably small step sizes. While the scanning step size was set to 150 $\mathrm{nm}$ in our analyses in order to cover wide sample areas, a primary electron beam with a diameter of approximately 20 $\mathrm{nm}$ and a comparatively small precession angle of $0.15^{\circ}$ were used for our analyses.

Due to the comparatively small precession angle, the virtual bright field (Fig. 5a) and crystallite orientation (Fig. 5b) maps contain thin film buckling artifacts. The crystallite orientation map shows nevertheless sufficient pixel value homogeneity throughout many of the crystallites.

Note that the "microscope's vacuum area" is clearly visible at the top of both the virtual bright field and the dark field maps, Fig. 6. This area is labeled (and appears "light orange") in the crystallite orientation map, Fig. 5b. Virtual bright field images are maps of the intensity of the transmitted electron beam at each scanning increment. The "software equivalent" of an aperture is used to remove the diffracted beams. The numbers in round brackets in Fig. 5a identify individual crystallites that are correspondingly labeled in the virtual dark field maps in the right column of Fig. 6.
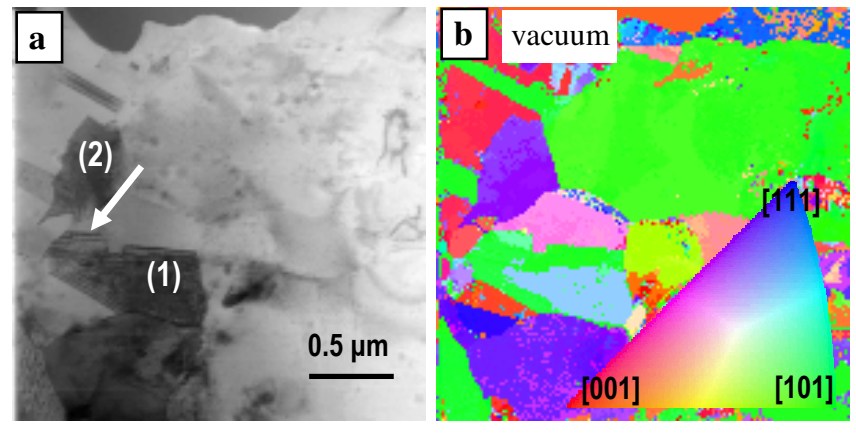

Fig. 5 Crystallite orientation mapping of polycrystalline copper, $0.15^{\circ}$ precession angle. (a) Virtual bright field map, (b) crystallite orientation map with the $[001] /($ red) - [101]/(green) - [111]/(blue) stereographic triangle for FCC crystallites as inset.

Virtual dark field images of individual crystallites (or crystallite areas) are obtained with the ASTAR software by a click of the mouse after a suitable "software aperture" has been set around a particular reflection spot in one of the automatically recorded electron diffraction patterns from that crystallite. The positions of such apertures are indicated in the diffraction patterns in the left column of Fig. 6. The corresponding virtual dark field map shows higher intensity for that particular crystallite and also for all of the various sample areas that were simultaneously oriented in reflection conditions, e.g. Fig. 6 right column.

The experimental electron diffraction spot patterns that formed the basis for the crystallite mapping process, on the other hand, can be displayed by clicking with the mouse on a crystallite in either the virtual bright field map or the 
crystallite orientation map. In the left column of Fig. 6, two such patterns are shown.

Due to the comparatively small precession angle, resemblances of "dynamical diffraction contrast" are present in the virtual bright field map, Fig. 5a, and the virtual dark field maps, right column of Fig. 6. Such a contrast feature is marked by arrows in both figures. While thickness fringes of classical dynamical diffraction contrast are a sequence of dark and bright stripes at a crystallite edge in the virtual bright field map of the marked crystallite, the contrast is reversed in the respective virtual dark field map.
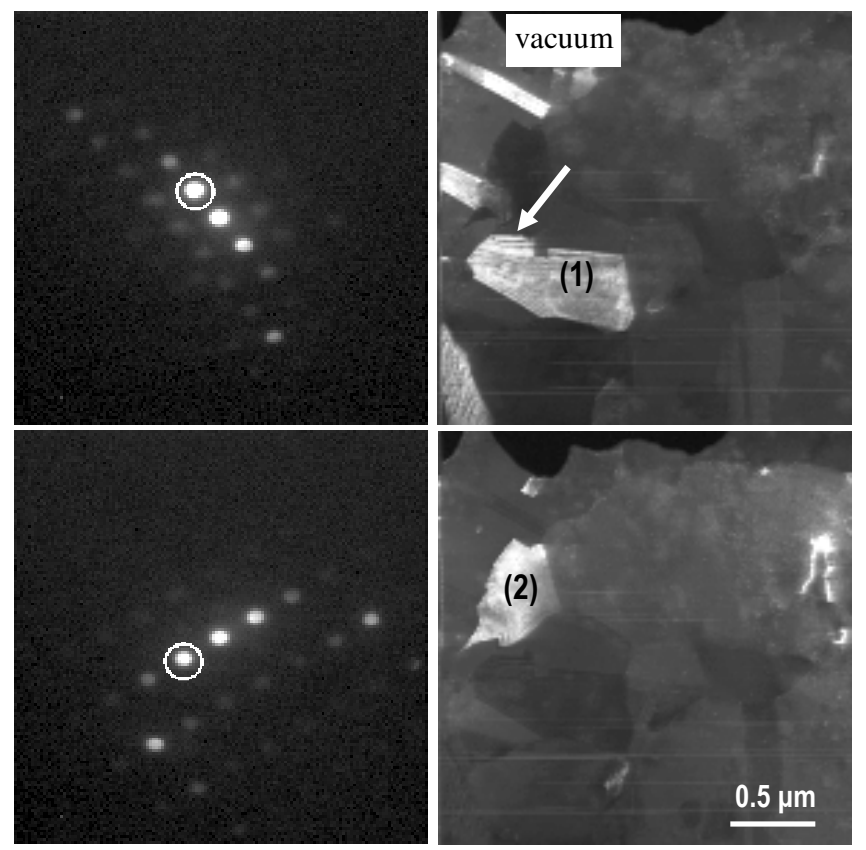

Fig. 6 Crystallite orientation mapping of polycrystalline copper, $0.15^{\circ}$ precession angle. Left column: Experimental diffraction spot patterns of selected crystallites. Right column: Corresponding virtual dark field maps.

This kind of quasi-classical diffraction contrast at low precession angles will have its usage in more thorough offline analyses of polycrystalline materials. Larger precession angles, on the other hand, tend to average such contrasts out but limit the maximal obtainable orientation resolution (as mentioned above). A precession angle of $0.6^{\circ}$ was, e.g., sufficient to suppress diffraction contrasts in a bright field tomography study of $\mathrm{Sn}$ precipitates in an $\mathrm{Al}$ matrix [11].

\section{PHASE IDENTIFICATION AND ORIENTATION DISTRIBUTION OF SELF ASSEMBLED CRYSTALLITES IN GALLIUMARSENIDE}

At the above mentioned JEOL JEM 2200FS of Humboldt University Berlin, the data for the maps of "self assembled surface contactable - partially embedded" MnAs rich crystallites in a GaAs matrix, were collected at $200 \mathrm{kV}$ from a cross section, Fig. 7, and a plan view sample, Figs. 8 and 9, see also refs. [3,9]. A PED device ASTAR/DigiSTAR from NanoMEGAS is interfaced to this microscope and it serves as demonstration site for this company for middle Europe.

Both the scanning-precession step size and the probe size were on the order of magnitude $20 \mathrm{~nm}$ for the cross section sample (but can be set to much smaller values when needed).
While there was no precession during the recording of the data for the maps in Figs. 7a and 7b, a precession angle of $1^{\circ}$ was utilized for the recording of the data in Figs. 7c and 7d.

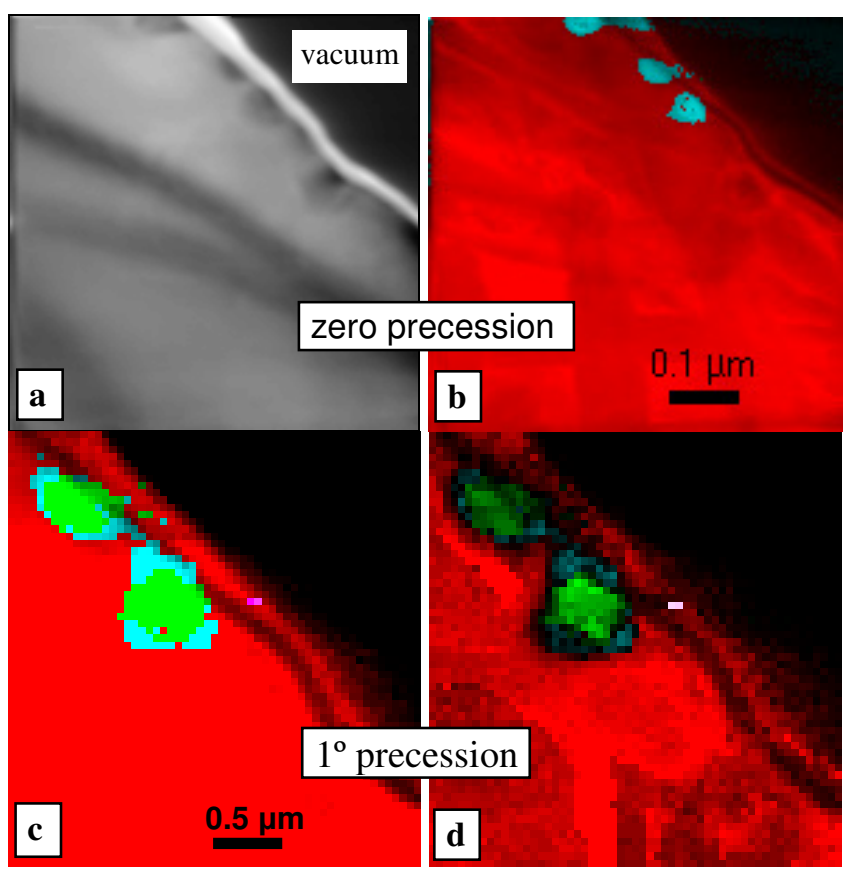

Fig. 7 Crystallite phase mapping of MnAs rich crystallites in a GaAs matrix, [110] cross sections, to illustrate advantages of using (scanning) PED data in comparison to "on optical axis" (scanning) transmitted beam data. (a) Virtual bright field and (b) crystallite phase map overlaid with the corresponding correlation index map from the same specimen area; both for zero precession. (c) Crystallite phase map overlaid with the corresponding correlation index map and (d) crystallite phase map overlaid with both the correlation index map and the phase reliability index map for a precession angle of $1^{\circ}$ from the upper right part of the specimen area of (a) and (b). The color code in (b) to (d) is: GaAs appears in red, $\beta$-MnAs in light blue, and $\alpha-\mathrm{MnAs}$ in green.

Note that there are at least two possible candidate phases for the partly embedded crystallites: the ferromagnetic $\alpha$ MnAs with space group $\mathrm{P}_{3} / \mathrm{mmc}$ and the paramagnetic $\beta$ MnAs with space group Pnma. If the crystallites were (Mn,Ga)As mixed crystals with atomic ordering, there could be many more candidate phases which the crystallites could possibly possess.

Figure $7 \mathrm{~b}$ shows the distribution of three $\beta$-MnAs crystallites (light blue) in the GaAs matrix (red). The cross correlation map is superimposed on this crystallite/matrix phase map. Due to the zero-precession angle, the cross correlation indices are generally low in good correspondence to the thin film buckling that is visible in the virtual bright field map of Fig. 7a.

Precession at an angle of $1^{\circ}$ enhanced the cross correlation indices everywhere, see Fig. 7c, and the thin film buckling effect was effectively removed. The cross correlation index map is also superimposed on the crystallite/matrix phase map of Fig. 7c, allowing for a direct assessment of the effect of PED data on the accuracy of the method. (All of these indices are defined in ref. [3]). Note also that comparatively a larger area is mapped in Figs. $7 \mathrm{a}$ and $7 \mathrm{~b}$ that in Figs. 7c 
and $7 \mathrm{~d}$. The mapped area in Figs. $7 \mathrm{c}$ and $7 \mathrm{~d}$ contains only the two lower MnAs rich crystallites of Fig. 7b.

The "distribution" of two possible MnAs phases is shown in Fig. 7c in light blue for the paramagnetic orthorhombic $\beta$ phase and green for the ferromagnetic hexagonal $\alpha$-phase on the red background of the GaAs matrix. Figure $7 \mathrm{~d}$ shows finally the superposition of the map in Fig. 7c with the corresponding phase reliability map. Since the phase reliability is low for both MnAs phases, the study of this cross section sample remained somewhat inconclusive. This might perhaps be due to the MnAs rich crystallites being fully embedded in the GaAs matrix in the direction of the transmitted electron beam.

Complementary results were obtained for this materials system in plan view, where the free surface of the MnAs rich crystallites is in the direction of the transmitted electron beam, Figs. 8 and 9. The scanning-precessing step size of the primary electron beam was for these experiments set to $5 \mathrm{~nm}$. The full widths at half maximum of the primary electron beam was approximately $2.4 \mathrm{~nm}$ and a precession angle of $0.9^{\circ}$ was utilized. While Fig. 8 shows the phase reliability index map and the MnAs crystallite/GaAs matrix phase map, three crystallite orientation maps are shown in Fig. 9.

The maps in Fig. 8 demonstrate that essentially all of the identified MnAs crystallites may possess the orthorhombic crystal phase. The small "blue area" of the MnAs crystallite that has been marked by a black arrow in Fig. 8d is not proof that hexagonal MnAs should be present. The corresponding crystallite orientation maps of Fig. 9 were, therefore, only calculated for the two phase system orthorhombic MnAs and GaAs.
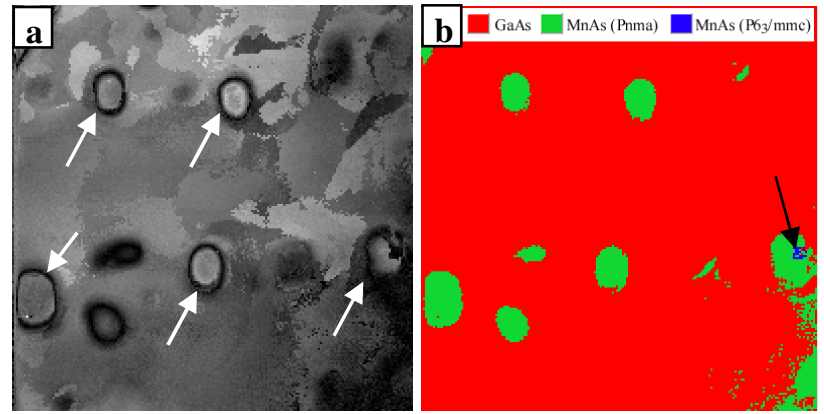

Fig. 8 Crystallite phase mapping of MnAs rich crystallites in a GaAs matrix, [001] plan view, $0.9^{\circ}$ precession angle. (a) Phase reliability index, and (b) crystallite/matrix phase map of both orthorhombic MnAs (in green) and hexagonal MnAs (in blue) on a (red) GaAs background. Note that the outlines of phase boundaries around five larger MnAs rich crystallites (that are marked by white arrows) are clearly visible in the phase reliability map. The black arrow in (b) is explained in the text

Figure $9 \mathrm{a}$ to $9 \mathrm{c}$ indicate that at least the "five larger" MnAs (rich) crystallites (as marked by arrows in Fig. 8a) are well ordered/textured in all three spatial dimensions, i.e. in two mutually perpendicular directions that are within the thin sample film and in the direction that is parallel to the GaAs wafer normal. Figure 10 shows finally ordinary TEM bright field images of MnAs rich crystallites in a GaAs wafer. The surface roughness of the sample is clearly revealed in the cross section, Fig. 10a.
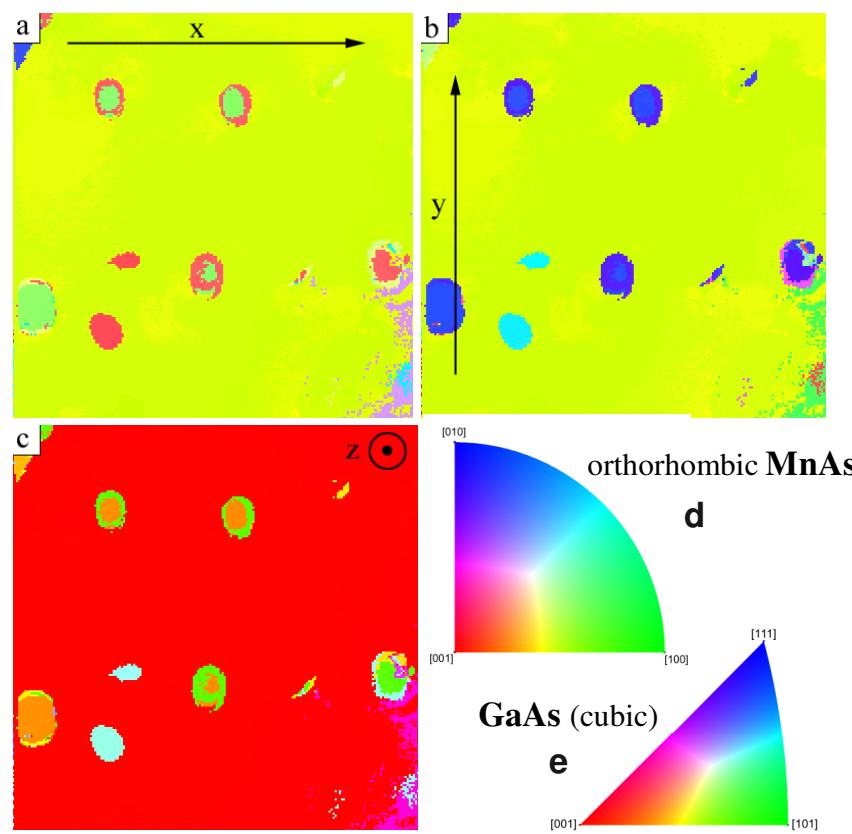

Fig. 9 Crystallite orientation mapping of orthorhombic MnAs in a GaAs matrix, [001] plan view, $0.9^{\circ}$ precession angle, same sample area as in Fig. 8. The GaAs orientations in both (a) and (b) are mutually perpendicular and belong to the (001) zone of the matrix. The red "background" color in (c) signifies the [001] orientation of the GaAs matrix. In the second row of this figure, (d) represents the color code of these maps for GaAs and (e) for the orthorhombic MnAs crystallites, respectively.
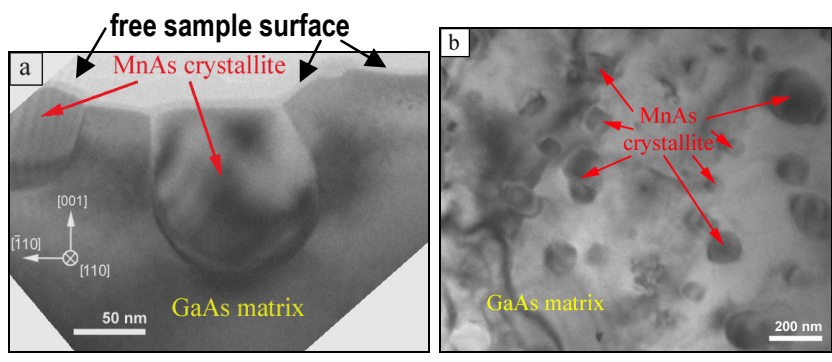

Fig. 10 Ordinary TEM bright field images of self assembled, surface contactable, partially embedded MnAs crystallites in a GaAs matrix (on a (001) GaAs wafer). (a) [110] cross section and (b) [001] plan view.

The nature of the identification process of crystallite phases by cross correlations of their electron diffraction spot patterns with templates that are calculated from candidate structures sets limits to the accuracy of the mapping processes. Obviously, these processes can only be as accurate as the presumed prior knowledge about the crystallite phases is. If there are a wide variety of candidate crystallite phases, the recommendation is simply to calculate maps for exhaustive combinations of these candidates and to make good usage of the corresponding phase reliability maps. In order to ensure correct crystallite phase identifications, it will also be worthwhile to look at a multitude of individual PED spot patterns (such as obtainable straightforwardly for individual crystallites in the maps from the automatically recorded data) and to employ hierarchal structural fingerprinting strategies such as outlined in refs. [4-6]. 


\section{OPEN ACCESS CRYSTALlOGRAPHIC DATABASES}

The required prior crystallographic knowledge about a sample that is necessary for the crystallite phase identification by the ASTAR software is just what is needed to calculate electron diffraction spot pattern templates in the kinematic approximation. As an alternative to the well known commercial databases, one may use comprehensive open-access databases. More than 170,000 comprehensive datasets are currently available for inorganic crystals at the combined web sites of the Crystallography Open Database (COD) [12], the COD's mainly inorganic and educational subsets [13], the American Mineralogist Crystal Structure Database [14], and the former Linus Pauling File [15].

Note that the COD has currently more than 143,000 entries and is rapidly growing [16]. All of its entries can be visualized interactively at the atomic level at one of the two Portland/Oregon based website mirrors [17]. All datasets of the COD are in the form of Crystallographic Information Files (CIFs) [18], i.e. the preferred format for the electronic dissemination of crystallographic data as endorsed by the International Union of Crystallography [18].

\section{SUMMARY AND CONCLUSIONS}

The basics of precession electron diffraction in a transmission electron microscope were briefly discussed in the first section of this paper. An automated system for the mapping of nanocrystal phases and orientations in a TEM was subsequently described. This system is primarily based on the projected reciprocal lattice geometry as extracted from experimental electron diffraction spot patterns and benefits greatly from the precession diffraction mode. Comprehensive open-access crystallographic databases may be used in support of the automated crystallite phase identification process by the system's software and were, therefore, also briefly mentioned.

We believe that the automated mapping technique in the transmission electron microscope is capable of addressing materials science problems for which there are no solutions by the well known electron backscattering based mapping techniques of scanning electron microscopy. Our briefly mentioned analysis of preferred orientations of self assembled - partially embedded - surface contactable MnAs rich crystallites in a GaAs matrix could well be such an example.

\section{ACKNOWLEDGMENTS}

This research was generously supported by the Oregon Nanoscience and Microtechnologies Institute, the Army Research Laboratories, Portland State University, and the "Nachwuchsakademie MatWerk" of the Deutsche Forschungsgemeinschaft (DFG). Peter Moeck is grateful for the hospitality during his sabbatical stay at Wolfgang Neumann's former research group/Chair for Crystallography at the Humboldt University of Berlin.

\section{REFERENCES}

[1] E. Rauch, M. Véron, J. Portillo, D. Bultreys, Y. Maniette, and S. Nicolopoulos, "Automatic Crystal Orientation and Phase Mapping in TEM by Precession Diffraction”, Microscopy and Analysis, Issue 93, pp. S5-S8, November 2008.

[2] R. Vincent and P. Midgley, "Double conical beam-rocking system for measurement of integrated electron diffraction intensities", Ultramicroscopy, vol. 53, pp. 271-282, 1994.

[3] P. Moeck, S. Rouvimov, E. F. Rauch, M. Véron, H. Kirmse, I. Häusler, W. Neumann, D. Bultreys, Y. Maniette, and S. Nicolopoulos, "High spatial resolution semi-automatic crystallite orientation and phase mapping of nanocrystals in transmission electron microscopes", Cryst. Res. Technol., vol. 46, pp. 589-606, 2011.

[4] P. Moeck and S. Rouvimov, "Precession electron diffraction and its advantages for structural fingerprinting in the transmission electron microscope", Z. Kristallogr., vol. 225, pp. 110-124, 2010.

[5] P. Moeck and S. Rouvimov, "Structural fingerprinting of nanocrystals in the transmission electron microscope: utilizing information on projected reciprocal lattice geometry, 2D symmetry, and structure factors", In: Drug Delivery Nanoparticles Formulation and Characterization, Informa, 2009 (Drugs and the Pharmaceutical Sciences, vol. 191), pp. 270-313.

[6] P. Moeck and P. Fraundorf, "Structural fingerprinting in the transmission electron microscope: overview and opportunities to implement enhanced strategies for nanocrystal identification", Zeits. Kristallogr., vol. 222, pp. 634-645, 2007; expanded version in open access at: arXiv:0706.2021.

[7] E. F. Rauch, J. Portillo, S. Nicolopoulos, D. Bultreys, S. Rouvimov, and P. Moeck, "Automated nanocrystal orientation and phase mapping in the transmission electron microscope on the basis of precession electron diffraction", Zeits. Kristallogr. vol. 225, pp. 103-109, 2010.

[8] P. Moeck, S. Rouvimov, E. F. Rauch, and S. Nicolopoulos, "Structural Fingerprinting of Nanocrystals: Advantages of Precession Electron Diffraction, Automated Crystallite Orientation and Phase Maps", In: Electron Crystallography for Materials Research and Quantitative Characterization of Nanostructured Materials, (Eds.: P. Moeck, S. Hovmöller, S. Nicolopoulos, S. Rouvimov, V. Petkov, M. Gateshki, and P. Fraundorf), Mater. Res. Soc. Symp. Proc. vol. 1184, pp. 49-60, 2009; ISBN: 978-1-60511-157-5.

[9] I. Haeusler, S. Nicolopoulos, E. F. Rauch, K. Volz, and W. Neumann, "Crystallite phase and orientation mapping of MnAs in GaAs on the basis of automatically analyzed precession electron diffraction spot patterns", Proc. IMC17, Rio de Janeiro.

[10] G. Benner, H. Niebel, and G. Pavia, "Nano beam diffraction and precession in an energy filtered Cs corrected transmission electron microscope", Cryst. Res. Technol., vol. 46, pp. 580-588, 2011.

[11] J. M. Rebled, L. Yedra, J. Portillo, S. Estradé and F. Peiró, "Combination of Electron Tomography and Electron Beam Precession applied to Sn precipitates in Al matrix", Proc. IMC17, Rio de Janeiro.

[12] http://cod.ibt.lt (in Lithuania), http://www.crystallography.net and http://cod.ensicaen.fr/ (in France), http://qiserver.ugr.es/cod (in Spain), http://nanocrystallography.org and http://nanocrystallography.net (in Oregon, USA).

[13] http://nanocrystallography.research.pdx.edu/CIF-searchable/, data on some 20,000 crystals.

[14] http://rruff.geo.arizona.edu/AMS/amcsd.php, data on some 10,000 minerals.

[15] http://crystdb.nims.go.jp, data on some 30,000 metals and alloys.

[16] S. Gražulis, D. Chateigner, R. T. Downs, A. F. T. Yokochi, M. Quirós, L. Lutterotti, E. Manakova, J. Butkus, P. Moeck, and A. Le Bail, "Crystallography Open Database - an open-access collection of crystal structures “, J. Appl. Cryst. vol. 42, pp. 726-729, 2009; open access: http://journals.iucr.org/j/issues/2009/04/00/kk5039/kk5039.pdf.

[17] http://jcod.nanocrystallography.net.

[18] S. Hall and B. McMahon, International Tables for Crystallography, vol. G, Springer, 2005; supporting open access papers on the usage of CIF at: http://www.iucr.org/resources/cif/documentation. 